prolonged, intrauterine asphyxia in the term infant. According to Volpe JJ (Neurology of the Newborn, 2nd ed, Philadelphia, Saunders, 1987), the localization of hypoxic-ischemic encephalopathy in the infant is generally more diffuse than in asphyxiated animals, and selective brainstem injury is rare and frequently fatal. (See Progress in Pediatric Neurology I. PNB Publishers, 1991;p334).

\title{
EEG IN DIAGNOSIS OF PERIVENTRICULAR LEUKOMALACIA
}

The specificity and sensitivity of positive rolandic sharp waves (PRSW) on serial EEGs for the diagnosis of periventricular leukomalacia (PVL) in premature infants was studied in 83 newborn cases (11\% of 765 premature infants) treated at the Hopital Antoine Beclere, Assistance Publique/Hopitaux de Paris, Clamart, France. All prematures underwent repeated ultrasound scanning and EEGs during the first weeks of life. Those showing persistent hyperechoic periventricular densities on ultrasound had MRIs performed. Of the 83 with PVL, 65 had cystic PVL. PRSW were present in 55 cases, and EEG abnormalities preceded the ultrasonic detection of cystic PVL. PRSW were specific markers for both cystic and noncystic PVL, and PRSW sensitivity was dependent on gestation age, with higher frequency $(88 \%)$ in infants of $28-32$ weeks than in those born before 28 weeks (32\%). (Baud O, d'Allest A-M, Lacaze-Masmonteil T et al. The early diagnosis of periventricular leukomalacia in premature infants with positive rolandic sharp waves on serial electroencephalography. I Pediatr May 1998;132:813-817). (Reprints: Anne-Marie d'Allest MD, Service de Reanimation et Pediatrie Neonatales, Hopital Antoine Beclere, Assistance Publique/Hopitaux de Paris, 157, rue de la Porte de Trivaux, 92 141 Clamart, France).

COMMENT. Positive rolandic sharp waves on the EEG are specific for the development of periventricular leukomalacia and subsequent neurologic impairment in premature infants.

Outcome predictive value of neonatal assessment of preterm infants. Clinical assessment in the intensive-care unit was used to predict normal or abnormal motor outcome at 6 years, in a study of 153 infants followed in the Department of Perinatal Medicine, King George V Hospital, Sydney, Australia (Lacey JL, Henderson-Smart DJ. Assessment of preterm infants in the intensivecare unit to predict cerebral palsy and motor outcome at 6 years. Dev Med Child Neurol 1998;40:310-318). Neonates were classified according to the absence, transient occurrence, or persistence of atypical signs, including jitters, asymmetric tonic neck reflex, hypotonia, or hypertonia. Absence of atypical signs predicted normal development for $62 \%$ of 116 , whereas one or more atypical features predicted major motor dysfunction in 7 of 11 infants.

Retinopathy of prematurity and autistic disorder. Blindness due to retinopathy of prematurity was strongly associated with autistic spectrum disorders in a controlled population-based study of 27 children at the University of Stockholm, Sweden (Ek U et al. Relation between blindness due to retinopathy of prematurity and autistic spectrum disorders: a population-based study. Dev Med Child Neurol 1998;40:297-301). The association was probably mediated by brain damage, independent of the blindness per se.

\section{SCOLIOSIS IN SPASTIC CEREBRAL PALSY}

The natural history and risk factors for progressive severe scoliosis were evaluated in 37 institutionalized spastic cerebral palsy patients followed at the Department of Orthopaedic Surgery, Shinshu University School of Medicine, Matsumoto, and at Centers in Nagano, Japan. Scoliosis started before age 10 yrs and 
progressed rapidly during the growth period, reaching a $60^{\circ}$ magnitude by 17 yrs. Those with scoliosis $>60^{\circ}$ were bedridden and had thoracolumbar curves. Risk factors for progression of scoliosis in spastic cerebral palsy are: a spinal curve of $40^{\circ}$ before age $15 \mathrm{yrs}$; total body involvement; being bedridden; a thoracolumbar curve. Early surgical intervention to prevent progression is recommended in patients with these risk factors. (Saito N, Ebara S, Ohotsuka K, Kumeta H, Takaoka K. Natural history of scoliosisi in spastic cerebral palsy. Lancet June 6, 1998;351:1687-1692). (Respond: Dr Naoto Saito, Dept of Orthopaedic Surgery, Shinshu University School of Medicine, Asahi 3-1-1, Matsumoto 3900-8621, Japan).

COMMENT. Reports indicate that bracing is frequently ineffective for scoliosis with cerebral palsy and surgery is advocated in severe cases. Fusion is intended to facilitate sitting and standing, improve pulmonary and upper limb function, and benefit nursing procedures. The above study provides guidelines for early surgical intervention.

\section{METABOLIC DISORDERS}

\section{FOCAL NEUROLOGIC DEFICITS WITH HYPOGLYCEMIA}

Clinical manifestations and outcome of transient focal neurologic deficits (TFND) associated with hypoglycemia were evaluated in 44 children with insulindependent diabetes mellitus observed retrospectively during a 5 year period at the University of Trieste, Italy. Symptoms included transient hemiparesis in 54 episodes, usually during sleep, and alternating right- and left-sided in 3 ; aphasia in 16 episodes; and preceded by a brief convulsion in 8 episodes. Duration of episodes was $<2$ hrs in $30,2-12 \mathrm{hrs}$ in 13 , and $>12 \mathrm{hrs}$ in 2 . Hypoglycemia was documented in 26 , and in 18 of these episodes, symptoms did not resolve promptly after sugar administration. The long-term outcome was benign, no patient having persistent neurologic abnormalities and none developing migraine at follow up. Invasive tests were not considered mandatory. (Pocecco M, Ronfani L, and Italian Collaborative Paediatric Diabetologic Group. Transient focal neurologic deficits asociated with hypoglycemia in children with insulin-dependent diabetes mellitus. Acta Paediatr May 1998;87:542-544). (Respond: Dr M Pocecco, Department of Paediatrics, Children's Hospital "Burlo Garofolo," University of Trieste, Italy).

COMMENT: Transient focal neurologic deficits in children with diabetes are often but not invariably asociated with hypoglycemia and their long-term outcome is good. Alternative causes for an episodic hemiparesis in diabetes include vascular spasm, hemiplegic migraine, and Todd's paresis.

\section{COGNITIVE, MOTOR, AND BEHAVIORAL FUNCTION IN PKU}

Measures of cognitive, frontal lobe (executive), behavioral and motor function were administered to 18 children (aged 12-101 months) with phenylketonuria followed at the University of Rochester School of Medicine, NY. Current phenylalanine levels were within recommended range (120-485 $\mathrm{mcmol} / 1$ ) in 65\%, and lifetime levels ranged from 206-1331 (mean, 499). "Individual variation" (SD of lifetime level) ranged from 76-547 (av, 270). Lower current phenylalanine (PHA) levels were associated with higher cognitive functioning in children older than 3 yrs. Higher current and average levels correlated with more difficult temperament on behavior scales. Motor function was impaired in PKU children with current PHA levels above $360 \mathrm{mcmol} / 1$, and lower motor scores tended to correlate with older age and current PHA levels. 